Editorial, Acta Neurologica Scandinavica:

Ferraro D, Guicciardi C, De Biasi S, Pinti M, Bedin R, Camera V, Vitetta F, Nasi M, Meletti S,

Sola P: Plasma neurofilaments correlate with disability in progressive multiple sclerosis patients. Acta Neurol Scand 2019, in press.

\title{
Plasma neurofilament light in progressive multiple sclerosis
}

Henrik Zetterberg ${ }^{\mathrm{a}, \mathrm{b}, \mathrm{c}, \mathrm{d} *}$

${ }^{a}$ Department of Psychiatry and Neurochemistry, Institute of Neuroscience and Physiology, the Sahlgrenska Academy at the University of Gothenburg, Mölndal, Sweden

${ }^{b}$ Clinical Neurochemistry Laboratory, Sahlgrenska University Hospital, Mölndal, Sweden

cDepartment of Neurodegenerative Disease, UCL Institute of Neurology, Queen Square, London, $U K$

${ }^{d} U K$ Dementia Research Institute at UCL, London, UK

*Correspondence:

Henrik Zetterberg, $M D, P h D$

Clinical Neurochemistry Laboratory

Sahlgrenska University Hospital

S-43180 Mölndal

Tel: +46313430025

Fax: +4631419289

E-mail: henrik.zetterberg@clinchem.gu.se

Keywords: Neurofilament light; plasma; biomarker; multiple sclerosis; disability

Neurofilaments (Nfs) are a family of neuronal intermediate filaments involved in the growth and stability of axons, and, through incorporation into different supramolecular assemblies, also in synaptic organization and function in the central nervous system. Pioneering work by Lars

Rosengren and colleagues in the late 1980s led to the establishment of an enzyme-linked immunosorbent assay (ELISA) based on polyclonal antisera that allowed for the reliable quantification of the neurofilament light (NfL) subunit in cerebrospinal fluid (CSF). ${ }^{1,2}$ The utility of CSF NfL as an axonal injury marker was first proven in amyotrophic lateral sclerosis, ${ }^{3}$ but it

This article has been accepted for publication and undergone full peer review but has not been through the copyediting, typesetting, pagination and proofreading process, which may lead to differences between this version and the Version of Record. Please cite this article as doi: 10.1111/ANE.13184

This article is protected by copyright. All rights reserved 
soon became clear that NfL is a general marker of neuroaxonal injury, irrespective of cause, as verified in recent meta-analyses. ${ }^{4,5}$

The first version of the assay was gradually improved in regards to analytical sensitivity and is now based on monoclonal antibodies against the mid-domain of the protein (the so-called rod region). ${ }^{6,7}$ In 2015, the most widely used ELISA was transferred onto the Single molecule array (Simoa) platform, ${ }^{8}$ which is a bead-based digital ELISA that allows for single molecule measurements, leading to a broad dynamic range. ${ }^{9}$ This improved analytical sensitivity made it possible to measure NfL concentration in regular serum or plasma samples. ${ }^{8}$ Importantly, using Simoa, the correlation of serum with CSF NfL levels is high. ${ }^{10}$ Given the robust relationship between CSF and blood NfL concentrations, it has been proposed that blood NfL could replace CSF NfL for the assessment of ongoing axonal injury in CNS diseases. However, it remains unclear if blood NfL concentrations change concurrently with CSF without delay, and if this correlation remains strong across a longitudinal trajectory, which is an important consideration for an early marker of neurodegeneration or monitoring therapeutic response. A recent cautionary paper suggests that plasma NfL may be less sensitive as an endpoint than CSF NfL in clinical trials. ${ }^{11}$ Another potential confounder is the degree of peripheral nerve damage influencing blood NfL levels; patients with Charcot-Marie-Tooth disease have significantly increased plasma NfL concentration compared with age-matched control individuals. ${ }^{12}$

In recurrent remitting multiple sclerosis (RRMS), CSF NfL concentration correlates with imaging evidence of white matter injury, degree of disability, disease activity, and time since last relapse. ${ }^{13}$ Further, it has been shown that CSF NfL normalizes in response to disease-modifying treatments (DMTs). For example, MS patients starting natalizumab, a DMT with high efficacy, experienced a normalization of their CSF NfL levels down to those seen in healthy controls within 6-12 months. ${ }^{14}$ With the new, ultrasensitive NfL assay, this result has been corroborated further. Similar to CSF NfL, it has been shown that serum NfL levels in MS patients are independently associated with disease activity and that DMT reduces serum NfL levels, in many cases down to the levels seen in age-matched healthy controls. ${ }^{15,16}$ Repeated NfL measurements in peripheral blood for detecting axonal damage may thus represent a new possibility in MS monitoring. 
In progressive MS, more research on the value of CSF NfL is needed. CSF NfL concentration is increased in progressive MS and reduced in response to immunosuppressive therapy, ${ }^{17}$ but robust relationships to disability progression has not yet been established. In the current issue of Acta Neurologica Scandinavica, Ferraro et al. set out to examine the association of plasma NfL concentration with disability, disease severity and disability progression in 70 untreated patients with primary or secondary progressive MS using RRMS patients $(n=21)$ and healthy controls $(\mathrm{n}=10)$ as contrast groups. ${ }^{18}$ The data show that plasma NfL concentration was significantly higher in progressive MS compared with RRMS and the healthy control group in a manner that correlated with Expanded Disability Status Scale and MS Severity Score. Nine progressive MS patients had repeated plasma NfL concentrations determined over 2 years and all of these increased in NfL more than what could be expected by normal ageing.

Altogether, the study speaks for the usefulness of plasma NfL as a biomarker for disease activity and progression also in progressive MS. Hopefully, this simple blood test could be used to speed up the evaluation of novel candidate drugs against progressive MS in clinical trials.

\section{Disclosures}

HZ has served at scientific advisory boards for Wave, Samumed, CogRx and Roche Diagnostics, has given lectures in symposia sponsored by Biogen and Alzecure, and is a co-founder of Brain Biomarker Solutions in Gothenburg AB, a GU Ventures-based platform company at the University of Gothenburg.

\section{References}

1. Karlsson JE, Rosengren LE, Haglid KG. A rapid HPLC method to separate the triplet proteins of neurofilament. J Neurochem. 1987;49(5):1375-1378.

2. Karlsson JE, Rosengren LE, Haglid KG. Polyclonal antisera to the individual neurofilament triplet proteins: a characterization using ELISA and immunoblotting. $J$ Neurochem. 1989;53(3):759-765.

3. Rosengren LE, Karlsson JE, Karlsson JO, Persson LI, Wikkelso C. Patients with amyotrophic lateral sclerosis and other neurodegenerative diseases have increased levels of neurofilament protein in CSF. J Neurochem. 1996;67(5):2013-2018. 
4. Gaetani L, Blennow K, Calabresi P, Di Filippo M, Parnetti L, Zetterberg H. Neurofilament light chain as a biomarker in neurological disorders. J Neurol Neurosurg Psychiatry. 2019;90(8):870-881.

5. Bridel C, van Wieringen WN, Zetterberg H, et al. Diagnostic Value of Cerebrospinal Fluid Neurofilament Light Protein in Neurology: A Systematic Review and Meta-analysis. JAMA Neurol. 2019.

6. Norgren N, Karlsson JE, Rosengren L, Stigbrand T. Monoclonal antibodies selective for low molecular weight neurofilaments. Hybrid Hybridomics. 2002;21(1):53-59.

7. Norgren N, Rosengren L, Stigbrand T. Elevated neurofilament levels in neurological diseases. Brain Res. 2003;987(1):25-31.

8. Gisslen M, Price RW, Andreasson U, et al. Plasma Concentration of the Neurofilament Light Protein (NFL) is a Biomarker of CNS Injury in HIV Infection: A Cross-Sectional Study. EBioMedicine. 2016;3:135-140.

9. Andreasson U, Blennow K, Zetterberg H. Update on ultrasensitive technologies to facilitate research on blood biomarkers for central nervous system disorders. Alzheimers Dement (Amst). 2016;3:98-102.

10. Kuhle J, Barro C, Andreasson U, et al. Comparison of three analytical platforms for quantification of the neurofilament light chain in blood samples: ELISA, electrochemiluminescence immunoassay and Simoa. Clin Chem Lab Med. 2016;54(10):1655-1661.

11. de Flon P, Laurell K, Sundstrom P, et al. Comparison of plasma and cerebrospinal fluid neurofilament light in a multiple sclerosis trial. Acta Neurol Scand. 2019;139(5):462-468.

12. Sandelius A, Zetterberg H, Blennow K, et al. Plasma neurofilament light chain concentration in the inherited peripheral neuropathies. Neurology. 2018;90(6):e518-e524.

13. Khalil M, Teunissen CE, Otto M, et al. Neurofilaments as biomarkers in neurological disorders. Nat Rev Neurol. 2018;14(10):577-589.

14. Gunnarsson M, Malmestrom C, Axelsson M, et al. Axonal damage in relapsing multiple sclerosis is markedly reduced by natalizumab. Ann Neurol. 2011;69(1):83-89.

15. Novakova L, Zetterberg H, Sundstrom P, et al. Monitoring disease activity in multiple sclerosis using serum neurofilament light protein. Neurology. 2017;89(22):2230-2237.

16. Disanto G, Barro C, Benkert P, et al. Serum Neurofilament light: A biomarker of neuronal damage in multiple sclerosis. Ann Neurol. 2017;81(6):857-870. 
17. Axelsson M, Malmestrom C, Gunnarsson M, et al. Immunosuppressive therapy reduces axonal damage in progressive multiple sclerosis. Mult Scler. 2014;20(1):43-50.

18. Ferraro D, Guicciardi C, De Biasi S, et al. Plasma neurofilaments correlate with disability in progressive multiple sclerosis patients. Acta Neurol Scand. 2019. 